\title{
Moderate prenatal alcohol exposure was associated with a modest increase in adolescent learning and behavioural difficulties
}

\author{
Olson HC, Streissguth AP, Sampson PD, et al. Association of prenatal alcohol exposure with behavioral and learning problems in \\ early adolescence.J Am Acad Child Adolesc Psychiatry 1997 Sep;36:1187-94.
}

\section{Objective}

To determine whether an association exists between prenatal alcohol exposure and behavioural and learning difficulties in early adolescence.

\section{Design}

Population based cohort study from the Seattle Longitudinal Prospective Study on Alcohol and Pregnancy.

\section{Setting \\ USA.}

\section{Participants}

250 infants of women who reported social alcohol use during pregnancy and 250 infants of women who reported infrequent or no alcohol use during pregnancy were selected in 1974-5 and followed up to a mean age of 14.5 years. Mothers were predominantly white, married, middle class, $20-30$ years of age, and at low risk for adverse pregnancy outcomes. 249 boys and 215 girls completed the 14 year follow up.

\section{Assessment of risk factors}

Mothers were interviewed during the fifth month of pregnancy about early and mid-pregnancy alcohol use, demographics, pregnancy history, nutrition, and use of caffeine, tobacco, and illicit and prescription drugs. Net prenatal alcohol exposure was assigned a composite score based on the weighted average of 13 consumption scores. $78 \%$ of mothers reported some drinking during mid-pregnancy. The median consumption for a drinker was approximately 1 half ounce drink of alcohol per day before recognition of pregnancy and less than a quarter ounce of alcohol per day during mid-pregnancy. "Drinkers" ingested an average of 2 to 2.5 drinks per occasion before pregnancy recognition and less than 2 drinks per occasion during mid-pregnancy. 39\% of mothers reported at least one instance of "binge" drinking
( $\geqslant 5$ drinks per occasion) early in pregnancy and 24\% reported this during mid-pregnancy.

\section{Main outcome measures}

At the 14 year follow up, data on adolescent learning and behaviour were collected by adolescent self report (questionnaires given by a research examiner during a $4 \mathrm{~h}$ clinic session), parent report (structured telephone interviews and posted Child Behaviour Checklist), and research examiner report (Behaviour Rating Form based on the $4 \mathrm{~h}$ of clinic testing). The researcher was unaware of the adolescent's prenatal alcohol exposure, previous test results, and home environment. Net learning and behaviour difficulty was assigned a composite score based on the weighted average of 186 items reported by the adolescent, parent, and research examiner.

\section{Main results}

The unadjusted correlation between prenatal alcohol exposure and adolescent difficulties was 0.31 . Increased alcohol dose was generally associated with increased difficulties; however, not all exposed adolescents had problems and associations were highly variable. Stepwise multiple regression techniques were used to adjust for child sex, maternal education, presence of 2 parents in the home at ages 4 and 14 years, and household smoking. After adjusting for covariates, the correlation between prenatal alcohol exposure and adolescent difficulties was 0.20 . There were no interactions between these covariates and prenatal alcohol exposure.

\section{Conclusion}

Moderate prenatal alcohol exposure was associated with a modest increase in adolescent learning and behavioural difficulties.

Sources of funding: National Institute on Alcohol Abuse and Alcoholism.

For article reprint:Dr C Olson, Fetal Alcohol and Drug Unit, 180 Nickerson, Suite 309, Seattle, WA 98109, USA. Fax +1 2066852903.

\section{Commentary}

This study by Olson $e t$ al addresses a relatively unexplored question: what is the relation of low dose prenatal alcohol exposure to adolescent adaptive and learning behaviours? There is no known safe amount of alcohol consumption during pregnancy, ${ }^{1}$ and longitudinal studies are critical to understanding the teratogenic effects of alcohol. In this well designed cohort study, the authors showed that adolescents at highest risk of behavioural and learning problems were prenatally exposed to alcohol and were exposed earlier in the pregnancy. Also consistent with previous findings, the results were highly variable among adolescents who were similarly exposed. ${ }^{1}$ Furthermore, the correlation between the net prenatal exposure score and the behavioural/learning difficulties score was weak. The weak finding may reflect a truly weak association because the prob- lems examined are likely of multifunctional origin. The low study power and likely misclassification of the approximately 200 problem variables which were examined may have contributed to attenuating the true correlation between prenatal alcohol exposure and the problems examined. The assessment of maternal alcohol use by self report once in mid-pregnancy could have resulted in misclassification of true consumption. None the less, the authors have addressed an important question and have reinforced the need for delineating alcohol specific outcomes, dose response effects, and the genetic or environmental factors that may explain the differential effects of prenatal alcohol exposure.

The study findings reinforce the importance of health education to promote alcohol abstention during pregnancy, as even moderate intake of alcohol may have deleterious effects. If further research finds a strong relation between alcohol and child/adolescent behaviours, it will be important to study the mechanisms underlying this relation. This information could help adolescent health nurses understand how alcohol related behavioural problems are manifested and, perhaps, distinct from problems with different aetiologies. A better understanding of the effects of alcohol could lead to specific assessment tools and intervention strategies to ameliorate them.

Wendy L Hellerstedt, MPH, PhD School of Public Health,

Maternal and Child Health Program University of Minnesota

Minneapolis, Minnesota, USA

1 Abel EL, Hannigan JH. Maternal risk factors in fetal alcohol syndrome: provocative and permissive influences. Neurtoxicol Teratol 1995;17:445-62. 